\title{
The effect of massage therapy on sleep quality in cardiac care unit patients
}

\author{
Arezoo Khosravi', Fariba Bolourchifard ${ }^{2 *}$, Mahnaz Ikhani ${ }^{3}$ and Mohamad Amin Pourhoseingholi ${ }^{4}$ \\ ${ }^{1}$ Master of Medical Surgical Nursing, Student Research Committee, School of Nursing and Midwifery, Shahid \\ Beheshti University of Medical Sciences, Tehran, Iran \\ ${ }^{2}$ PhD in Nursing, Assistant Professor, Department of Medical Surgical Nursing, School of Nursing and \\ Midwifery, Shahid Beheshti University of Medical Sciences, Tehran, Iran \\ ${ }^{3}$ Doctorate in Clinical Practice, Assistant Professor, Department of Medical Surgical Nursing, School of \\ Nursing and Midwifery, Shahid Beheshti University of Medical Sciences, Tehran, Iran \\ ${ }^{4} \mathrm{PhD}$ in Biostatistics, Associate professor, Gastroenterology and Liver Disease Research Center, Research \\ Institute for Gastroenterology and Liver Diseases, Shahid Beheshti University of Medical Sciences, Tehran, Iran
}

\section{ABSTRACT}

Low sleep quality is one of the most common complaints in patients admitted to CCU. This study aimed to determine the effect of Massage Therapy on Sleep Quality, admitted in the cardiac care unit. This research is a quasi-experimental study. 60 cardiac patients admitted to CCU at Shariati Hospital in Tehran were selected and randomly divided into two intervention and control groups. Members of the intervention group for a week, For one week, in 4 sessions, each session is 12 minutes twice a day, in the morning and in the evening or at least once before bed time were treated by massage, but the control group did not received the massage. Data collected by demographic characteristics questionnaire and Pittsburg sleep quality (PSQI) and were analyzed by using of statistical methods such as paired t-test and analysis of covariance. Results showed improved quality of sleep among the intervention group quality scores in the post-test significantly improved compared to pre-test $(\mathrm{P}=0 / 001)$ but in the control group was not observed significant difference between the pre-test and post-test $(\mathrm{P}=0 / 520)$. Also, there was a significant difference between post-test score in two groups of intervention and control with control of pre-test effects $(\mathrm{P}=0 / 001)$. Therefore, massage therapy has improved the quality of sleep in the intervention group. According to effectiveness of massage therapy on sleeping quality in patients hospitalized in the coronary care unit, this method can be used to reduce undesirable effects of decreased sleep quality in patients.

KEY WORDS: MASSAGE THERAPY, SLEEPING QUALITY, CARDIOVASCULAR PATIENTS, CARDIAC CARE UNIT

\section{ARTICLE INFORMATION:}

*Corresponding Author: bolourchifard@gmail.com, bolourchi@sbmu.ac.ir

Received $12^{\text {th }}$ Oct, 2017

Accepted after revision $21^{\text {st }}$ Dec, 2017

BBRC Print ISSN: 0974-6455

Online ISSN: 2321-4007 CODEN: USA BBRCBA

a Thomson Reuters ISI ESC and Crossref Indexed Journal -. NAAS Journal Score 2017: 4.31 Cosmos IF: 4.006

- A Society of Science and Nature Publication, 2017. All rights reserved.

Online Contents Available at: http//www.bbrc.in/

DOI: $10.21786 / \mathrm{bbrc} / 10.4 / 6$ 


\section{INTRODUCTION}

Cardiovascular diseases have the highest mortality rates and will continue to be the leading cause of death in the world by 2020 (Shafie et al., 2013). At present, cardiovascular disease accounts for 38\% of i the world (Moller, 2010), and the first cause of death in Iran. It has also been anticipated that, by 2030, the order of causes of death would be included ischemic heart disease, cerebro-vascular diseases, AIDS and chronic pulmonary diseases (Azizi, 2008).In spite of increasing awareness of cardiovascular diseases prevention and planning which governments have done in this regard, and given the aging population, the prevalence of cardiovascular diseases, and consequently the need for Coronary care unit (CCU) and cardiac intensive care beds are on the rise. As many as 2940 people across the country need to be admitted to cardiac care units (Talebi et al., 2009). This indicates an increase in the need for hospitalization and the number of beds in the cardiac care unit in Iran. Meanwhile, almost every disease with significant pain or discomfort, such as respiratory, cardiovascular, digestive and neurological diseases, can negatively affect sleep quality (Jahne et al., 2012). Sleep problems in patients with heart failure are more prevalent than those without this disease, and factors such as respiratory problems, increased age, medication, anxiety and depression play a significant role in this (Suna, 2015).

Sleep is a regular, repeatable, and reversible physiologic event in which a person experiences a decrease in consciousness, a relative loss of skeletal muscle (volitional) and a significant increase in the threshold of response to external stimuli. More than a third of human life span is spent in sleep. Therefore, any intermittent hypoxia and disorder in quantity, quality or pattern of sleep can have a significant negative effect on the person's physical and mental health and lead to the development of cardiovascular complications (Wang, 2010). For example, Gvstafsn writes on the findings of his studies: trouble in falling asleep is an independent risk factor for cardiac events in men. He also believes that there is a link between inadequate sleep and many clinical manifestations of coronary artery disease such as angina, cardiac arrhythmia, increased blood pressure, respiratory problems, the risk of developing myocardial infarction and sudden death (Bayley, 2010). Sleep also affects the cardiovascular system regulation, so that at the time of awakening the heart of a healthy person, on average, 70 to 80 beats per minute, while at bedtime it is reduced to 60 times per minute. Find (Fontana and Pittiglio, 2010). Conversely, sleep deprivation increases heart rate and increases the myocardial need for oxygen (Matthews et al., 2010).

Nerbass et al. (2010) found that although coronary artery bypass graft surgery is a common operation (prac- tice) with a low mortality rate and relieves angina symptoms in a desirable manner, but recovery from post-cardiac surgery is followed by symptoms and signs of pain and psychological distress, and sleep problems. Considering all the emphases and warnings about the effects of sleep deprivation in hospitalized patients, especially in cardiac care units, many of the patients admitted to these units experience problems caused by sleep and rest disturbances (Zolfaghari et al., 2013), and sleep disorders in patients admitted to intensive care units are highly prevalent (Habibzade et al., 2011). Although sleep problems can be somewhat controlled by medication, but due to the problems and complications of drug therapy (Cinder, 2007), the use of non-medical methods that can reduce sleep problems in cardiovascular patients is logical. In order to solve these problems, various nursing practices have been used as complementary therapies to help patients to meet their psychological and physical needs that among which massage therapy is an effective nursing intervention in relaxing, reducing stress, relaxing the mind and body in patients (Oshundi et al, 2013).

After a preliminary study on cardiac patients, Cutshall and colleagues suggested that massage be used as a complementary therapy to help reduce pain and anxiety in these patients. Nelson et al. (2008) found that massage with release of endorphins prevents from the transmission of pain messages and Wilkinson (2009) suggests that relaxation and eliminating anxiety can be due to reduced muscle spasm and thus reduce pain (Watson, 2011). Also, Castro and colleagues reported during their research that massage therapy in patients with fibromyalgia via decreased muscle tendon restriction, reduces anxiety and improves sleep quality and physical performance in these patients. Some research has shown that massage therapy is effective in improving sleep quality and reducing fatigue in patients during the recovery period after coronary artery bypass graft surgery (Narbass et al., 2010).

Kavehia et al. (2013) investigating the effects of massage therapy on psychological outcomes in post-cardiac surgery patients stated that massage therapy is effective in reducing pain and improving psychological outcomes in patients undergoing cardiac surgery. Massage therapy with parasympathetic stimulation can lead to effects such as lowering heart rate, reducing respiration, facilitating and returning to normal conditions in cardiovascular patients (Morskaa et al., 2010). Therefore, considering the low sleep quality of patients admitted to CCU, the complications of sleep medications, the need to use complementary and simple therapies in nursing, safe and inexpensive methods, it was decided to conducting a study aimed to determine the effect of massage on the quality of sleep in the patients admitted to intensive cardiac units, a step toward helping these patients 
through nursing interventions is taken. For this purpose, the aim of this study was to evaluate the effect of massage therapy on the quality of sleep in patients admitted to cardiac care units (CCU).

\section{MATERIALS AND METHODS}

This research is a semi-experimental study with university ethics committee approval. 60 patients hospitalized in the cardiac care units of selected hospitals of Tehran University of Medical Sciences in 2016, according to inclusion of research (complete consciousness, aged 18 to 85 years, the lack of use of a variety of complementary therapies during the previous three months, non-use of sedative and narcotic drugs, healthy areas of massage, and permission from the doctor, lack of severe neuropathy, mental retardation, blindness and deafness, lack of history of arthritis, joint rheumatoid arthritis and joint disorders and absence of coagulation disorders and diabetes) were selected using convenience and purposeful sampling method and randomly even numbers were assigned in the experimental group and odd numbers assigned to control group.

The method of doing research was that the researcher received confirmation from the committee of research and graduate education and the Ethics Committee of Shahid Beheshti University of Tehran and obtaining a referral from the university and presenting it to the management and office of nursing at Shariati Hospital in Tehran, and obtaining permission after their introduction to the head nurse and the staff of the cardiac care unit and patients, the purpose of the research was explained to them. Before initiating massage therapy, the researcher first provided the patient and his or her environment for intervention, in such a way as to preserve the patient's privacy and not feel insecure. Then, the subjects completed the questionnaires. For non-literate students, a researcher or another family member read the content to the patient and the questionnaire was completed. For the intervention (experimental) group, in addition to the routine pharmaceutical and non-pharmaceutical methods of unit, Shiatsu massage was performed in the following way:

A total of six points and each point for two minutes were massaged. The points under massage proposed by an expert and acupressure specialist, included the point of ht7, the point at the radial and proximal angles relative to the wrist line at the plantar and tendon level of fifth finger flexor, point kidney 3 point and surface between the ankles and the achilles tendon in the horizontal direction, the anmien point at the angle between the mastoid and the mandible is located one centimeter directly above the hair extension and below the midline and posterior of the head to cover the important points of the control of insomnia (Fig. 1).

The massage of every point lasts for two minutes, which included one minute of vertical and direct pressure, which began with a very gentle pressure, and the pressure increased to some extent that the patient would report a pleasant sensation of diffusion of a stream or lightness, or that the therapist's nail is discolored due to pressure, or the patient feel uncomfortable with the increase in pressure, the process is continued. Then massage the point is done for one minute. At the time of acupressure, you need to focus on quiet breathing and fingertip pressure. This intervention lasted for one week in 4 sessions and 24 minutes per session twice daily in the morning and afternoon, or at least once before bedtime. After the intervention, the sleep quality questionnaire was completed again. Patients in the control group had all the conditions of the patients in the experimental group, but no intervention was provided for them, and patients received only routine pharmacological and non-pharmacological care and the questionnaire was completed on the first and last days by them.

The tools used in this study included demographic questions (age, gender, marital status, duration of illness, etc.), and the Pittsburgh Sleep Quality Index (PSQI) which has been designed by Bays et al. in 1989 to assess

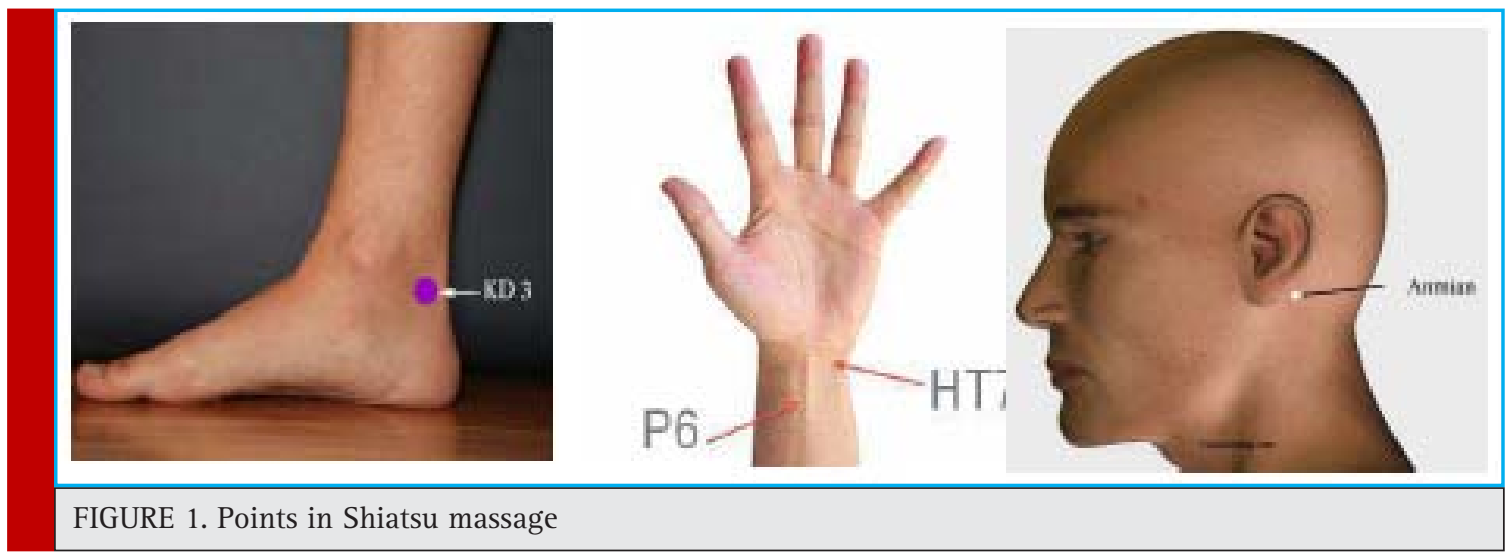


the quality of sleep in the psychiatric institution of Pittsburgh. This index consists of 19 questions in 7 dimensions (subjective sleep quality, sleep latency, sleep duration, habitual sleep efficiency, Sleep disturbances, use of sleeping medication and daytime dysfunction). Each part is scored from Zero (No problem) to 3 (there is a serious problem). The total score is between zero and 21, with higher scores indicating low sleep quality and vice versa. Bays et al. (1989) obtained internal consistency of the questionnaire using Cronbach's alpha of 0.83. In the Iranian version of this questionnaire, reliability was obtained by Cronbach's alpha of 0.46 and by the splithalf method of 0.52 (Heidari, Ehteshamzade and Marashi, 2010).

The software SPSS version 19 was used to analyze the data. Also, paired t-test was used to compare the mean of the groups. To compare the mean score of sleep quality before and after the intervention in two groups, covariance analysis was used. The significance level was considered as $\mathrm{p}<0.05$.

\section{RESULTS}

The mean age of the experimental group was 58.38 with a standard deviation of 19. 21 and the mean age of the control group 52.36 with a standard deviation of 17.55. The sample consists of 38 (63.33\%) males and 22 females (36.66\%) that each experimental and control group $(n=30)$ equally includes 19 males and 11 women. In the experimental group, $3(10 \%)$ were single and 27
(90\%) were married. In the control group, 4 (13.3\%) were single and 27 (86.7\%) were married. Finally, the mean duration of the disease was 3.30 months in the experimental group with a standard deviation of 6.25and in the control group, 5.48 months, with a standard deviation of 5.48 .

The mean and standard deviation of the pre-test and post-test of the studied variables in the intervention (experimental) and control groups are presented in Table 1.

As the results of the table above reveal, the post-test score of the intervention group was reduced by 2.93 in comparison with the pre-test score. In the intervention group, the post-test score increased by about 40\% compared to the pre-test score.

The calculated $\mathrm{Z}$ to evaluate the normal distribution of data for pre-test and post-test of the sleep quality score was 1.29 and 1.07, respectively, which was not statistically significant (Table 2). Therefore, parametric tests can be used to examine the research hypotheses.

Paired t-test was used to evaluate the effect of massage therapy on the quality of sleep in patients admitted in cardiac care units (Table 3).

As shown in table (3), the sleep quality score of the intervention group in the post-test has been decreased and according to the scoring of the questionnaire, the decrease in score means improving sleep quality. The $\mathrm{t}$-value calculated for changes in the mean in post-test of the intervention group compared to the pre-test score was 4.82 , which was statistically significant $(p=0.001)$. In contrast, the calculated $t$ value for the comparison of

Table 1. Mean and standard deviation of pre-test and post-test of sleep quality score
in two groups of intervention (experimental) and control
\begin{tabular}{|l|l|l|l|l|} 
variable & Group & Number & test periods & mean and standard deviation \\
\hline \multirow{4}{*}{$\begin{array}{l}\text { Sleep } \\
\text { quality }\end{array}$} & Intervention & \multirow{2}{*}{30} & Pre-test & $13.96(2.930$ \\
\cline { 4 - 5 } & & & Post-test & $11.03(1.27)$ \\
\cline { 4 - 5 } & \multirow{2}{*}{ control } & \multirow{2}{*}{30} & Pre-test & $13.06(2.75)$ \\
\cline { 4 - 5 } & & Post-test & $13.46(1.77)$ \\
\hline
\end{tabular}

\begin{tabular}{|l|l|l|l|}
\hline \multicolumn{4}{|l|}{ Table 2. Kolmogorov-Smirnov test for data normalization } \\
\hline Variable & Mean (SD) & Z Kolmogorov-Smirnov & p-value \\
\hline Pre-test score of sleep quality & $13.51(2.58)$ & 1.29 & 0.069 \\
\hline Post-test score of sleep quality & $12.25(1.96)$ & 1.07 & 0.20 \\
\hline
\end{tabular}

Table 3. Paired t-test results to examine the intra-group mean changes of intervention and control groups in the quality of life score

\begin{tabular}{|c|c|c|c|c|c|c|c|}
\hline \multirow[t]{2}{*}{ Variable } & \multirow[t]{2}{*}{ Group } & \multicolumn{2}{|c|}{ Pre-test } & \multicolumn{2}{|c|}{ Post-test } & \multirow[b]{2}{*}{$\mathrm{t}$} & \multirow[b]{2}{*}{ p-value } \\
\hline & & Mean & SD & Mean & SD & & \\
\hline & Intervention & 13.96 & 2.93 & 11.033 & 1.27 & 4.82 & 0.001 \\
\hline & Control & 13.06 & 2.75 & 13.46 & 1.77 & 0.64 & 0.52 \\
\hline
\end{tabular}


Table 4. One-way covariance analysis to compare the mean score of sleep quality in two intervention groups with control

\begin{tabular}{|l|l|l|l|l|l|l|l|l|}
\hline & Source & sum of squares & degree of freedom & mean square & F & P & eta coefficient & observed power \\
\hline \multirow{3}{*}{$\begin{array}{l}\text { Sleep } \\
\text { quality }\end{array}$} & Group & 83.07 & 1 & 83.07 & 34.56 & 0.001 & 0.377 & 0.99 \\
\cline { 2 - 9 } & Error & 137.003 & 57 & 2.40 & & & & \\
\cline { 2 - 9 } & Total & 9231.00 & 60 & & & & & \\
\hline
\end{tabular}

the pre-test and post-test mean of the control group was 0.64 , which was not statistically significant $(p=0.52)$.

To evaluate the consistency of variances between two groups, Levin test was used $(\mathrm{F}=0.73, \mathrm{df} 1=1,58=$ $\mathrm{df} 2, \mathrm{p}=0.08$ ). The insignificancy of Levine's test is that the variance in sleep quality score is identical in both groups and covariance can be used.

One-way covariance analysis was used to compare the mean of intervention and control groups. The results of one-way covariance analysis are presented in Table (4).

The results of one-way covariance analysis showed that the calculated $\mathrm{f}$ value for the comparison of the means in the intervention and control groups is 34.56 , which is statistically significant ( $p=0.001)$. Therefore, the results of this analysis showed that the intervention, namely, massage therapy, is effective on the quality of sleep in patients admitted to cardiac care units.

\section{DISCUSSION}

The aim of this study was to determine the effect of massage therapy on sleep quality of patients hospitalized in intensive care units. The findings showed that sleep quality in the intervention group improved after massage therapy. This finding is consistent with the results obtained in some previous studies. For example, Shafiee et al. (2013) examined the effects of massage therapy on the quality of sleep after surgery in patients undergoing coronary artery bypass graft surgery. The mean of quality of life scores in the experimental group and control group was $22.5 \pm 3.6$ and $22.3 \pm 3.8$, respectively. The difference was not statistically significant. After intervention, mean of quality of life scores of the patients in the experimental (intervention) group and control group was $5.5 \pm 4.7$ and $11 \pm 2.15$, respectively, which showed a significant difference $(\mathrm{p}<0.001)$. Also, the results showed that the use of massage therapy can improve the quality of sleep after surgery in patients undergoing coronary artery bypass graft surgery and considering the simplicity and low cost of this method, this method may be considered as a suitable supplement for medication and postoperative interventions in these patients. The differences between this study and the present study can be explained by the difference in the type of massage, the place where the work was carried out, the society and the research environment and the disease. The aim of this study was to investigate the effect of massage on the quality of sleep in patients with heart disease. In addition, the lack of improvement in sleep quality in the control group in the post-test compared with the pre-test is consistent with Arab and colleagues (2012), in a study entitled "the effect of acupressure on quality of life in patients undergoing hemodialysis".

Regarding the comparison of sleep quality of patients admitted to CCU in intervention and control groups, the findings showed that the intervention, namely, massage therapy, was effective on the quality of sleep in patients admitted to intensive care units. This finding is consistent with the study conducted by Narbas entitled "the effects of massage therapy on the quality of sleep after coronary artery bypass graft surgery. This research was performed on 57 patients undergoing coronary artery bypass surgery that were divided into two groups of control and massage group after discharge from the intensive care unit. Participants in the control group and the massage group were three nights without massage and three nights under the massage therapy. Patients were evaluated the next morning. The results of the study in the experimental group showed that sleep quality in the intervention group has been increased.

Some of the theories that look at massage offer assumptions (hypotheses) about the effectiveness of this method. For example, according to the gate control theory of pain, this method has been shown to increase the secretion of endorphins and enkephalins, thereby controlling pain, as well as improving the function of the immune system and eliminating toxins from the body. Also, based on the theory of nerve impulses, massage can inhibiting the afferent nervous messages and closure of nerve valve on the posterior horn of the spinal cord, inhibits the transmission of pain. It seems that by massage, the pituitary and hypothalamus glands are stimulated and endorphin is secreted as an intravenous narcotic similar to morphine by them, thus these neural mediators reduce the pain (Rigi et al., 2015).

Other researchers also believe that increased blood supply (increased intake of food, oxygen, and removal of cellular waste) and sensitization of muscles in relation to neural waves, of direct effects of massage therapy on the body. On the other hand, this technology can indirectly affect fatigue by reducing pain, improving depression and relaxation (Domingos \&t Barg, 2015). Massage reduces anxiety and tension, relieves pain and causes 
physical relaxation, leading to two-way energy transfer between the patient and the therapist, and as a general manipulation of the soft tissues of the body for restoring the metabolic balance of these tissues is used. The shortterm use of massage for hands, feet, neck and shoulders can have therapeutic effects. But many massage therapists focus on foot massage because of the lack of enough time to massage the whole body, which has the benefits of physical and mental relaxation, reducing anxiety and improving sleep (Shabani et al., 2005).In general, based on the literature on the effects of massage therapy, it seems that the effect of this treatment on the quality of sleep can be the direct and indirect effects of massage therapy. Its direct effects are due to changes in the hormonal and neuro-transmitter, and its indirect effects are due to the reduction of some of the variables affecting the quality of sleep such as reduced fatigue, stress and ... as a result of massage therapy.

Each research has limitations that can influence its outcomes. Some of the limitations of this research are: 1) lack of complete control of disturbing variables such as personality, physical and psychological variables as well as social, economic and cultural variables; 2) lack of suitable research facilities such as research room, proper chair and other necessary facilities in the hospital and therapeutic center; and 3) the limited power of generalizing the results due to the limited research sample group.

It is suggested that in future researches the effectiveness of massage therapy on other groups of patients with problems in sleep quality and other variables such as stress and anxiety of heart patients be investigated. It is also suggested that the effectiveness of massage therapy on the quality of sleep is compared with other therapies that are available to improve the quality of sleep, so that therapies with greater efficacy can be used to improve sleep quality. In addition, teaching this method of treatment to nurses, using this treatment to improve the quality of sleep in patients admitted to intensive cardiac units and preventing the negative consequences of poor sleep, such as heart problems, are some of the practical suggestions of the present study.

\section{CONCLUSION}

According to the results of this study and the effect of massage therapy on the sleep quality of patients admitted to the cardiac care unit, this method can be used to reduce the adverse effects of decreased sleep quality in these patients.

\section{ACKNOWLEDEMENTS}

This article was part of a Master of Medical Surgical Nursing Thesis and approved research plan of Shahid
Beheshti University of Medical Sciences, Tehran, Iran. An acknowledgement comes to action of all patients of hospitals affiliated to Tehran Universities of Medical Sciences that assisted in this study.

\section{REFERENCES}

Azizi, F. 2009 Projections Of Global Mortality And Burden Of Disease In Iran And World. Journal Of Research In Medical Sciences. 32, No.4, 259-260

Bailey, L. 2010. Strategies For Decreasing Patient Anxiety In The Perioperative Setting. Aorn Journal, 92, 445-460.

Castro-Sánchez, A. M., Matarán-Peñarrocha, G. A., GraneroMolina, J., Aguilera-Manrique, G., Quesada-Rubio, J. M. \& Moreno-Lorenzo, C. 2011 Benefits Of Massage-Myofascial Release Therapy On Pain, Anxiety, Quality Of Sleep, Depression, And Quality Of Life In Patients With Fibromyalgia. Evidence-Based Complementary And Alternative Medicine, 2011.

Cutshall, S. M., Wentworth, L. J., Engen, D., Sundt, T. M., Kelly, R. F. \&t Bauer, B. A. 2010. Effect Of Massage Therapy On Pain, Anxiety, And Tensionin Cardiac Surgical Patients: A Pilot Study. Complementary Therapies In Clinical Practice, 16, 92-95.

Domingos, T. D. S. \&t Braga, E. M. 2015. Massage With Aromatherapy: Effectiveness On Anxiety Of Users With Personality Disorders In Psychiatric Hospitalization. Revista Da Escola De Enfermagem Da Usp, 49, 450-456.

Fontana, C. J. \&t Pittiglio, L. I. 2010. Sleep Deprivation Among Critical Care Patients. Critical Care Nursing Quarterly, 33, 75-81.

Habibzade, H., Khalkhali, H. \& Ghaneii, R. 2011. Study Of The Relationship Between Restless Legs Syndrome And Sleep Disturbance Among Patients In Critical Care Units. Journal of Critical Care Nursing, 4, 153-158.

Kavei, P., Ebadi, A., Moradian, S. T. \&t Rahimabadi, M. S. 2015. The Effect Of Massage Therapy On Psychological Outcomes In Patients After Cardiac Surgery: A Mini Review. International Journal Of Medical Reviews, 1.

Matthews, K. A., Strollo Jr, P. J., Hall, M., Mezick, E. J., Kamarck, T. W., Owens, J. F., Buysse, D. J. \& Reis, S. E. 2011. Associations Of Framingham Risk Score Profile And Coronary Artery Calcification With Sleep Characteristics In Middle-Aged Men And Women: Pittsburgh Sleepscore Study. Sleep, 34, 711-6.

Möller, C., Wetter, T. C., Köster, J. \&t Stiasny-Kolster, K. 2010. Differential Diagnosis Of Unpleasant Sensations In The Legs: Prevalence Of Restless Legs Syndrome In A Primary Care Population. Sleep Medicine, 11, 161-166.

Moraska, A., Pollini, R. A., Boulanger, K., Brooks, M. Z. \& Teitlebaum, L. 2010. Physiological Adjustments To Stress Measures Following Massage Therapy: A Review Of The Literature. Evidence-Based Complementary And Alternative Medicine, 7, 409-418.

Nelson, R. \& Coyle, C. 2009. Using Massage To Reduce Use Of Sedative-Hypnotic Drugs With Older Adults: A Brief Report From A Pilot Study. Journal Of Applied Gerontology. 
Nerbass, F. B., Feltrim, M. I. Z., Souza, S. A. D., Ykeda, D. S. \& Lorenzi-Filho, G. 2010. Effects Of Massage Therapy On Sleep Quality After Coronary Artery Bypass Graft Surgery. Clinics, 65, 1105-1110.

Oshvandi, K., Abdi, S., Karampourian, A., Moghimbaghi, A. \& Homayonfar, S. 2014. The Effect Of Foot Massageon Quality Of Sleep In Ischemic Heart Disease Patients Hospitalized In Ccu. Iran J Crit Care Nurs, 7, 66-73.

Rigi, F., Feizi, A., Amirian, Z., Nasdri, M. \& Salehi, S. 2015. [Effect Of Foot Reflexology Massage On Pain In Patients Undergoing Coronary Bypass Surgery (Persian)]. Journal of Anesthesiology And Pain, 5, 42-49.

Shaban, M., Haji Amiri, P. \&t Kahrari, S. 2005. Immediate Effect Of Foot Massage On Patients' Vital Signs In Intensive Care Unit. Hayat, 20, 71-9.

Shafiee, Z., Babaee, S., Nazari, A. \& Atashi, V. 2013. The effect of massage therapy on sleep quality of patients after coronary artery bypass graft operation. Iranian Journal of Cardiovascular Nursing, 2, 22-29.

Suna, J. M., Mudge, A., Stewart, I., Marquart, L., O'rourke, P. Et Scott, A. 2015. The Effect Of A Supervised Exercise Training Programme On Sleep Quality In Recently Discharged Heart Failure Patients. European Journal Of Cardiovascular Nursing, 14, 198-205.

Synder, K. 2007. Biomedical Ethics Reviews. Complementary And Alternative Medicine, Ethics, The Patient And Physician Humana Press.

Wang, A. T., Sundt, T. M., Cutshall, S. M. \&t Bauer, B. A. Massage Therapy After Cardiac Surgery. Seminars In Thoracic And Cardiovascular Surgery, 2010a. Elsevier, 225-229.

Zolfaghari, M., Farokhnezhad Afshar, P., Asadi Noghabi, A. A. Et Ajri Khameslou, M. 2013. Modification Of Environmental Factors On Quality Of Sleep Among Patients Admitted To Ccu. Journal Of Hayat, 18, 61-68. 